\title{
Chronic Itch-Scratch Behaviour is a Cognitive Phenomenon Mediated through Neuroendocrine Receptors
}

\author{
Chan Kam Tim Michael*1, Tong Kwun Wah ${ }^{2}$ and Hiu Kim Yuen ${ }^{2}$ \\ ${ }^{1}$ Specialist in Dermatology, Hong Kong SAR, China \\ ${ }^{2}$ Softmind Limited, Hong Kong SAR, China
}

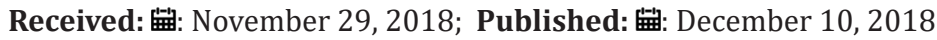

*Corresponding author: Chan Kam Tim Michael, Specialist in Dermatology, Hong Kong SAR, China

\begin{abstract}
Chronic itch is a common and severe debilitating disease in our population with a significant health burden. Pharmacological treatments despite widely used has not been satisfactory especially in managing anxiety and stress. Identification of a specific itch pathway involving the brain and its cognitive domains and functional anatomy provide opportunities to develop effective, non-invasive man machine devices to rehabilitate chronic pruritic dermatosis. In the following article, evidences and details of the chronic itch scratch cognitive pathways will be presented. A method devised to distract pruritic patients during scratching behaviour through analysing the electromyographic (EMG) signals from muscle sensors to predict the itch gesture. With the assistance of Apps functions; non-invasive signals like melody, music or virtual reality will be broadcasted and feedback in a timely manner.

Keywords : Itch-Scratch Behaviour; Anatomical Pathways; Itch Specific Neurones; Cognition; Behavioural Psychotherapy; Man-Machine Interaction

Abbreviations: EMG: Electromyographic; AD: Atopic Dermatitis; PN: Prurigo Nodularis; DA: Dermatitis Artefacta; MRSA: Methicillin Resistant Staphylococcus Aureus; CNS: Central Nervous System; CI: Central Nervous System; AI: Artificial Intelligence; PAR2: Protease-Activated Receptor 2; SLIGRL: Ser-Leu-Ile-Gly-Arg-Leu; DRG: Dorsal Root Of Ganglion; TRP1: Transient Receptor Potential vanilloid 1; TRA1: Transient Receptor Ankyrin 1; GRPC: Gastrin-Releasing Peptide Receptor; ET: Endothelin -1; NA: Noradrenaline; IFN-Y: Interferon-Y; SP: Substance P; NGF: Nerve Growth Factors; TSLP: Thymic Stromal Lymphopoietin; NPPB: Natriuretic Polypeptide B; F-MRI: Functional Magnetic Resonance Imaging; EMG: Electromyography; T-DCS: Transcranial Direct Current Stimulation; R-TMS: Repetitive Transcranial Magnetic Stimulation; MRGPRS: Mas-related G protein-coupled receptors
\end{abstract}

\section{Introduction}

Chronic pruritus is a distressing condition occurs both in childhood and adult with a prevalence between 8 to $38 \%$ of the population [1]. Scratching adversely affect a person's quality of life; sleep pattern, work concentration and social functions [2,3]. Stress, anxiety and depression may induce itch and vice versa [4, 5]. Chronic atopic dermatitis (AD), prurigo nodularis (PN), dermatitis artefacta (DA), psoriasis and uraemia are chronic dermatosis resistant to conventional therapy and diseases relapses are frequently seen. It constitutes a significant disease burden to health care system and community. Side effects of systemic medications are not uncommon and newer anti-inflammatory biologics are costly. The emergence of methicillin resistant Staphylococcus aureus (MRSA) complicated the management and further limit the present treatment protocol. There is a pressing need to formulate a more holistic, efficacious, non-invasive integrated approach to the management of chronic scratching. Advances in neuroscience provides important data to the existence of a specific itchy anatomical pathway comprising not only in the periphery; the skin but also the central nervous system (CNS) with its cognitive domains and anatomy [6].

The discoveries of itch specific neurones and the elucidation of such itch scratch cognitive behavioural pathway give insights and basis to develop newer psycho behaviour therapy involving manmachine interaction and artificial intelligence (AI). Achieving this may enable the person to rehabilitate to their normal physiological function of the sensation of itch and help restoring their normal body epidermal barrier function and immune status. The objectives of this article are to evaluate a possible central itch transmission anatomical functional pathway in human body relevant in chronic pruritic dermatosis based on Medline search and also to discuss possible non-pharmacological intervention strategies based on brain behaviour relationship. 


\section{Methods}

A Systematic Medline Search through PubMed published articles database involving the word "itch- scratch cycle, cognition".

\section{Results}

Altogether 126 articles were retrieved from the PubMed Medline published articles database involving the word "itchscratch cycle, cognition". Most articles focused on either the aberrant body immunity behind itch- scratch cycle in the dermis, epidermal barrier defects of the skin or the anti-pruritic effects of psychotropic drugs. There are approximately forty articles addressed the anti-pruritic effects of psychotropic medications; mainly the effects of antidepressants in recalcitrant difficult to treat pruritus [4]. One third of the articles outline systematically the anatomical pathways of itch from the peripheral nervous system through the spinal cords to the different cognitive areas of the brain. Less than ten percent of the searched articles focus on the brain cells, its circuitry and neurotransmitters in mediating itch. Majority of them are recent publications within last ten years. If we further narrowed down the search to the keywords: itch specific neurones, cognition; movement; no article was found.

In other words; most research efforts in the past concentrated on treating individual body compartment like the immune system, the skin epidermis and the pharmaco- psychological constituent of itch but neglecting a brain- centre top down approach on how cognition and different parts of the brain anatomy and circuitries systemically and mathematically propagating and intercepting the neurosensory electrical signals of itch and its pathology. Scratching movements essentially consists of a series of meteoric primitive cognitive reactions endowing a person to cope with stress, anxiety, fear and helplessness under adverse circumstances or hostile environmental conditions. Clarification and comprehending this complex central cognitive circuitry of itch and its mediators, new non- pharmacological, non-invasive psychological machineries may be innovated and made available to the sufferers in the near future.

\section{Peripheral Pathways and Mediators of Itch in the Skin}

Mas-related G protein-coupled receptors (Mrgprs) play a key role in mediating peripheral itch in the skin via specific itch afferent neurones to the spinal cord $[7,8]$. Mrgprs referred to a group of G-protein-coupled receptor (GPCR) located on neuronal cell surface act alone or synergistically with other pruritogenic receptors in transducing itch signals. Protease-activated receptor 2 (PAR2) is another GPCR important in mediating itch especially in defective epidermal barrier. A subpopulation of specific neurons belonged to the nociceptive $C$ fibers propagated the sensation of itch; not noxious painful stimuli; through $\mathrm{Ca}++$ influx depolarization $[9,10]$. MrgprA3, MrgprC11 and MrgprD+ expressed neurones located in the epidermis activated by Ser-Leu-Ile-Gly-Arg-Leu (SLIGRL) and $\beta$-alainine respectively relaying exclusive non-histaminergic itch to the dorsal root of ganglion (DRG) of the CNS [11]. On the other hand, MrgprA3 and MrgprC11 interact with Transient Receptor Potential vanilloid 1 (TRPV 1) and TRP ankyrin 1 (TRPA1) in the epidermis transmit histaminergic and non-histaminergic itchy signals to the CNS [12-14].
MrgprA3, MrgprC11 and MrgprD+ expressed afferent neurons penetrated in the epidermis together with TRPV 1 and TRPA 1 mediated itch signals from the peripheral skin synapses with gastrin-releasing peptide receptor (GRPC) interconnecting neurones in DRG [15]. Another itch mediator; endothelin -1 (ET1) was upregulated during scratching in patients in PN [16]. Recently; apart from voltage-gated calcium channel; voltage-gated sodium channel $(\mathrm{NaV}) 1.7$ was also found to be actively involved in mediating itch and pain triggered by spicy food [17].

\section{Neurogenic Inflammatory Cascades}

Persistent activation of the pruritogenic neuro pathways will result inflammatory cascades with the production of prostaglandins, bradykinin, substance P (SP), nerve growth factors (NGF), cytokines, insulin, serotonin, noradrenaline (NA), interferon- $\Upsilon$ (IFN- $\Upsilon$ ), histamine 1 to 4 , proteases and toll like receptors and its ligands by immunological activated mast cells, T lymphocytes, keratinocytes, granulocytes and macrophages [18]. These mediators will propagate pruritogenic depolarization signals; mainly through calcium influx; to the cerebral cortex through different synapses in the DRG. If the subjective sensation of itch conducted disinhibited; pathological scratchings and craving movements with its severe viscous consequences may be resulted. Elevated levels of Th2 cytokines interleukin - 31 (IL - 31) in particular are identified in-vitro and in-vivo to be important in exciting itch and scratch especially in AD. [19] IL - 31 was shown to cause severe pruritus in the malignant condition of Sezary syndrome [20]. IL-2, IL-4, IL-5, IL-10, IL-12, IL13 ad Thymic stromal lymphopoietin (TSLP) are other cytokines shown to be involved in the neurogenic inflammatory itch scratch inflammatory viscous cycle [21].

\section{Spinal Cord and the Central Itch Scratch Rewarding Circuitry}

Gastrin receptor proteins (GRP) and its receptors are the major gatekeeper of the pruritogenic signals in the DRG [15]. The neuropeptide; natriuretic polypeptide $\mathrm{b}(\mathrm{Nppb})$ expressed in a subset of TRPV 1 neurons found distributed widely in the spinal cord may interact with GRPC in inducing itch [22]. Interestingly, an interneurons Bhlhb5+ (B5-I) inhibits the transduction of itch through downregulation of TRP channels with the release of a kappa opioid receptor ligand neuropeptides called dynorphin; to impede itch [23]. A gate control mechanical itch and scratching was mediated by a subpopulation of spinal cord interneurons [24]. Spinal cord glial cells activation and reactive astrogliosis; abnormal increase of astrocytes; following the induction of the body inflammasome, toll -like receptor 4 (TLR-4) were also demonstrated during chronic itch $[25,26]$. The central station of itch transmission in our brain is the thalamus. The ascending spinothalamic tract relay histaminergic and non-histaminergic pruritogenic signals to the inner structures and cerebral cortex through the midbrain [27].

Hedonic scratch activated the primary somatosensory S1 areas of the cerebral cortex gave the perception of pleasantness and pleasure of itch in the cingulate cortex which decided the planned motor movement of scratching from the motor cortex [28,29]. The midbrain, ventral tegmental area, striatum, nucleus accumbens, 
caudate nucleus, ventromedial prefrontal cortex, insula and claustrum are all shown by functional magnetic resonance imaging (f-MRI) studies to be activated in this rewarding circuitry [29-33]. While this cerebral sensitization become unrestricted, pathological cravings and scratching behaviour surmounted as seen in obsessive compulsive disorders; pathological skin conditions like PN, DA and $\mathrm{AD}$ resulted [34]. Of particular concern, anxiety induced itch or vice versa; referred as the itch- anxiety cycle, showed intensive activities and signals in the hippocampus and adjacent anatomical structures involving the amygdala, the anterior cingulate cortex and insula cortex during itch and anxiety [35-37]. Others affective aspects of negative itch experience like depression and addiction are shown to be represented in subcallosal gray matter and nucleus accumbens of the brain.

On the inhibition side, different anatomical areas of the prefrontal cortex have been shown to be capable of sending hindering signals to amygdala to suppress fear, anxiety, stress and chronic itch via a top down regulation of cognition [4] Claustrum has been suggested to interocept and co-ordinate the conscious level and positively affect cognition especially in addictive behaviour. Finally, miswiring of various brain neurotransmitters like GABAs, serotonin, noradrenaline and dopamine has been implicated in mediating chronic itch in the brain. The diagrammatic representation of the itch-scratch brain cognitive pathway is illustrated in (Figure 1).

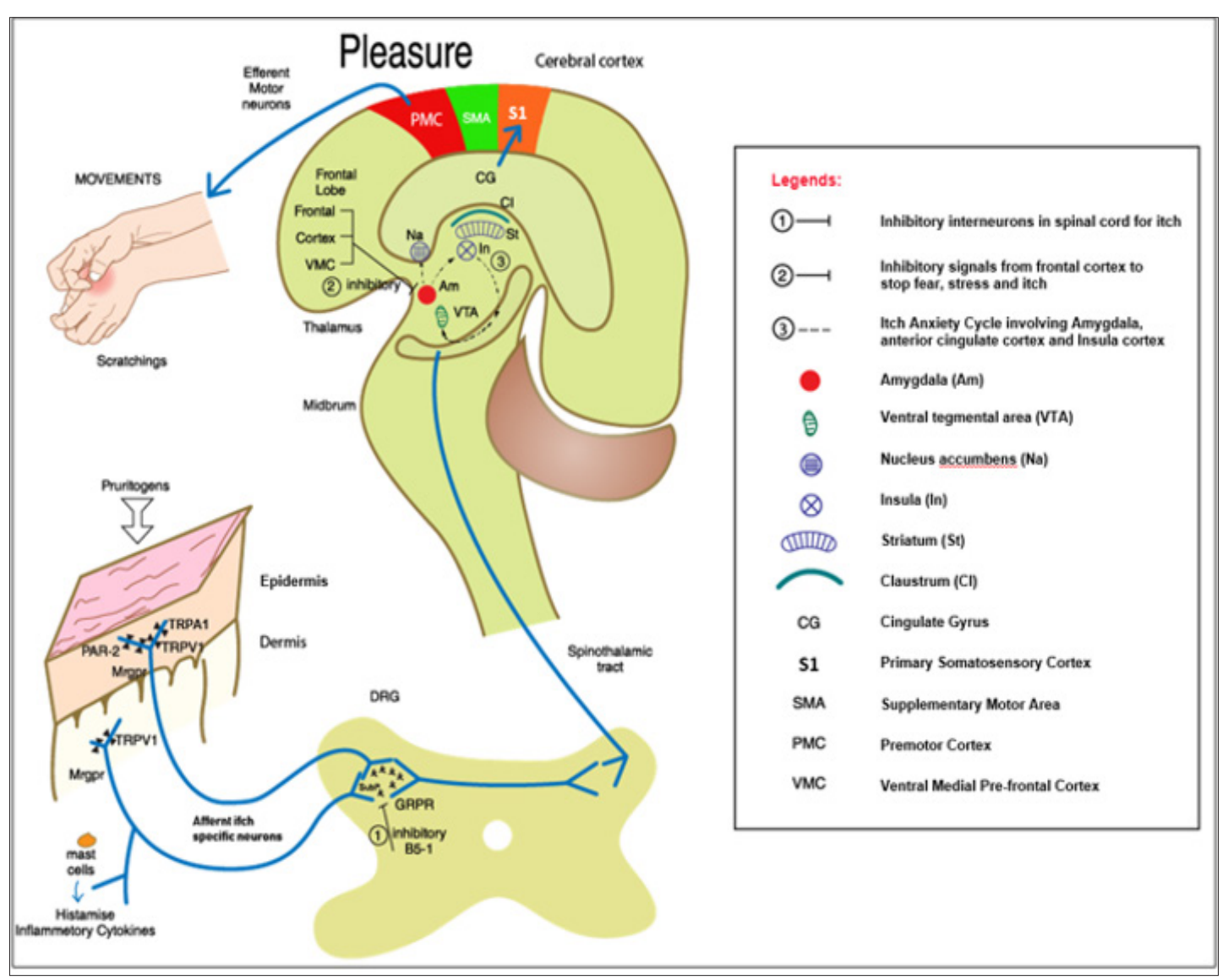

Figure 1: The diagrammatic representation of the itch-scratch brain cognitive pathway is illustrated.

\section{Non - Pharmacological Management of Chronic Itch}

Very few publications evaluate the psycho- neurological pathways of itch and cognitive functions. The cognitive involvement of the neuron specific itch pathway suggested more psychological cognitive behavioural therapy management initiatives in chronic pruritic conditions: patients and parents education, refocusing attention strategy on tasks unrelated to itch; virtual reality immersion and audio-visual distraction techniques; habit reversal training; arousal reduction and cognitive restructuring [38,39]. The authors have attempted to experiment a non-invasive sensor to a volunteer suffered from severe chronic $\mathrm{AD}$; resistant to conventional therapy; to detect scratching movement through electromyography (EMG). With the help of frequency analysis from Google deep Learning framework Tensorflow and wavelet transformations, relationships between the signals received from the electrodes setup on hand muscle have been identified. The detected interactions were visualised in a diagram which shows their relationships and respective intensity. The EMG signals from the muscle sensors to predict the itch gesture will then be analysed (Figure 2). 


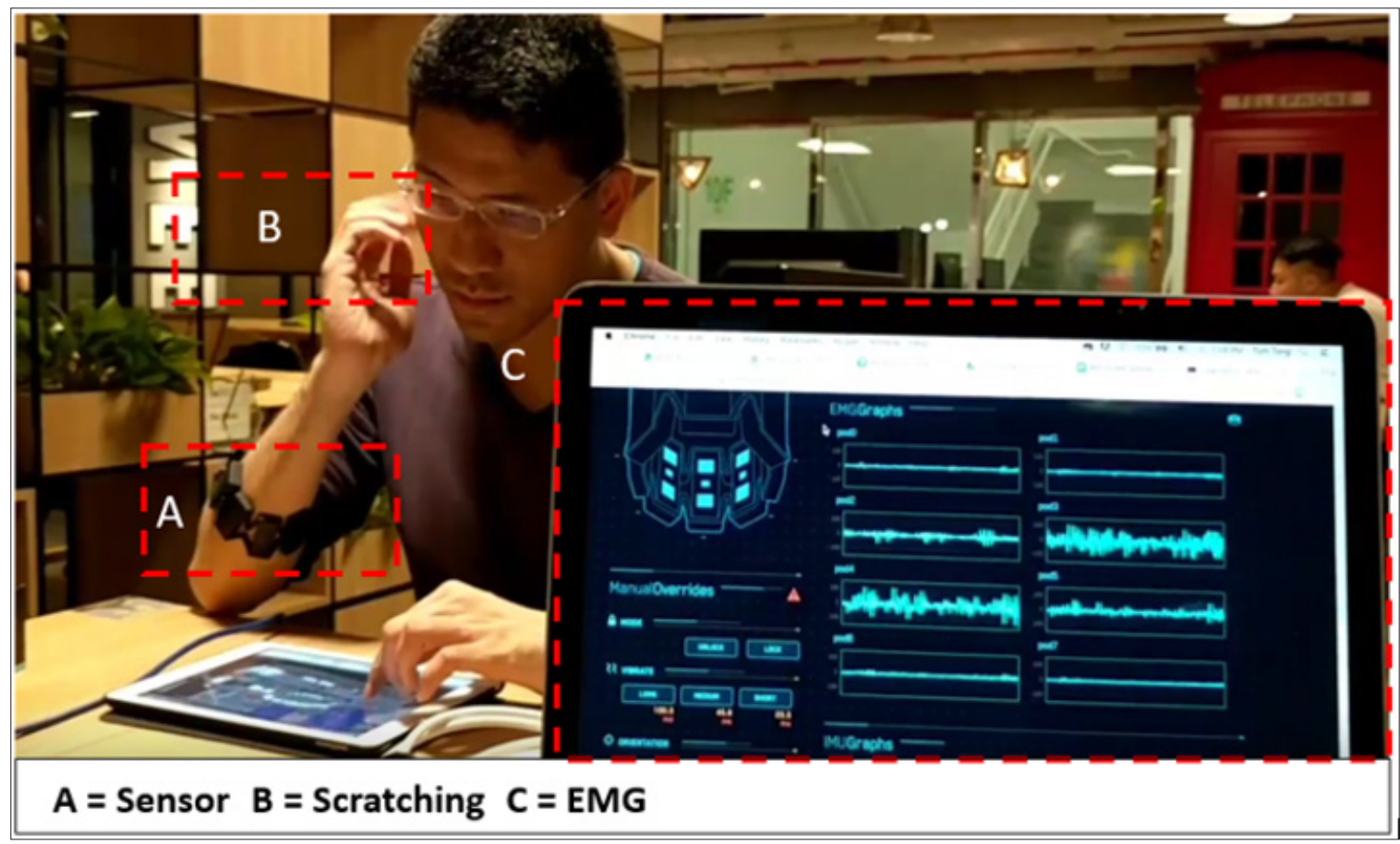

Figure 2: The EMG signals from the muscle sensors to predict the itch gesture will then be analysed.

Non-invasive signals like melody, music and virtual reality through Apps will be feedback to the patients in a timely manner. The recorded EMG patterns can be analysed, discussed, explained in-depths with the patients, their family members and correlated with attributes like intensity, life circumstances and events, time of the day, sleeping patterns and cognitive emotions. Emphasis should be placed on the acceptability of this machinery tools to the subject involved; non-invasiveness, user friendliness and low cost of the machine. Other interventions like transcranial Direct Current Stimulation (t-DCS) and repetitive Transcranial Magnetic Stimulation (r-TMS) are not available locally and their acceptability and potential adverse effects especially to children suffered from chronic pruritus has to be vigilant [40]. The validity of this kind manmachine interception of chronic itch must be validated in a more controlled manner with a larger population and prolonged period of careful clinical observation. Further researches are needed on this kind of man-machine interaction with the objective of enabling the chronic itch-scratching suffering victims to minimise stress, anxiety, fear, illness perception, sleep deprivation and depression of these distressing diseases.

\section{Conclusion}

In sum, pharmacological intervention to chronic pruritus and scratching is still the main paradigm in managing chronic resistant itchy dermatosis and widely practiced worldwide. Our research reveals that modern knowledges in neurosciences, cognitive imaging, motor behaviour and emotions give concrete evidence that itch - scratch - brain - cognition - movement axis exists. This not only enriches our understanding of the pathophysiology of chronic pruritic dermatosis but also provide opportunities in designing pharmacological and non-pharmacological machinery interventions. The best predictor of future perhaps is to create antiparadigm innovations especially in difficult to treat diseases.

\section{Acknowledgement}

The authors wish to thank Softmind Limited, Hong Kong SAR for providing technical support to this paper.

Part of the contents of this article will be presented orally in the coming conference on Movement and Cognition to be held at the Joseph B. Martin Conference Center of Harvard University's School of Medicine in Boston Massachusetts USA between 27-29 July 2018.

\section{References}

1. Mollanazar NK, Koch SD, Yosipovitch G (2015) Epidemiology of Chronic Pruritus: Where Have We Been and Where Are We Going? Curr Derm Rep 4(1): 20-29.

2. Chrostowska-Plak D, Reich A, Szepietowski JC (2013) Relationship between itch and psychological status of patients with atopic dermatitis. J Eur Acad Dermatol Venereol 27(2): e239-242.

3. Bender BG, Ballard R, Canono B, Murphy JR, Leung DY, et al. (2008) Disease severity, scratching, and sleep quality in patients with atopic dermatitis. J Am Acad Dermatol 58(3): 415-420.

4. Sanders KM, Akiyama T (2018) The vicious cycle of itch and anxiety. Neuroscience and Biobehavioral Reviews 87: 17-26.

5. Chida Y, Hamer M, Steptoe A (2008) A bidirectional relationship between psychosocial factors and atopic disorders: a systematic review and meta-analysis. Pyschosom Med 70(1): 102-116.

6. Sanders KM, Nattkemper LA, Yosipovitch G (2016) Advances in understanding itching and scratching: a new era of targeted treatments. 22(5): 2042. 
7. Liu Q, Tang Z, Surdenikova L, Kim S, Patel KN, et al. (2009) Sensory neuron-specific GPCR Mrgprs are itch receptors mediating chloroquineinduced pruritus. Cell 139(7): 1353-1365.

8. Qu L, Fan N, Ma C, Wang T, Han L, et al. (2014) Enhanced excitability of MRGPRA3- and MRGPRD-positive nociceptors in a model of inflammatory itch and pain. Brain 137(4):1039-1050.

9. Han L, Ma C, Liu Q, Weng HJ, Cui Y, et al. (2013) A subpopulation of nociceptors specifically linked to itch. Nat Neurosci 16(2): 174-182.

10. Wooten M, Weng HJ, Hartke TV, Borzan J, Klein AH, et al. (2014) Three functionally distinct classes of C-fibre nociceptors in primates. Nat Commun 20(5): 4122.

11. Liu Q, Sikand P, Ma C, Tang Z, Han L, et al. (2012) Mechanisms of itch evoked by $\beta$-alanine. J Neurosci 32(42): 14532-14537.

12. Wilson SR, Gerhold KA, Bifolck-Fisher A, Liu Q, Patel K, et al. (2011) TRPA1 is required for histamine-independent, Mas-related G proteincoupled receptor-mediated itch. Nat Neurosci 14(5): 595-602.

13. Wilson SR, Nelson AM, Batia L, Morita T, Estandian D, et al. (2013) The ion channel TRPA1 is required for chronic itch. J Neurosci 33(22): 92839294.

14. Shim WS, Tak MH, Lee MH, Kim M, Kim M, et al. (2007) TRPV1 mediates histamine-induced itching via the activation of phospholipase A 2 and 12-lipoxygenase. J Neurosci 27(9): 2331-2337.

15. Sun YG, Chen ZF (2007) A gastrin-releasing peptide receptor mediates the itch sensation in the spinal cord. Nature 448(7154): 700-703.

16. Kido-Nakahara M, Buddenkotte J, Kempkes C, Ikoma A, Cevikbas F, et al. (2014) Neural peptidase endothelin-converting enzyme 1 regulates endothelin 1-induced pruritus. J Clin Invest 124(6): 2683-2695.

17. Devigili G, Eleopra R, Pierro T, Lombardi R, Rinaldo S, et al. (2014) Paroxysmal itch caused by gain-of-function Nav1.7 mutation. Pain 155(9): 1702-1707.

18. Geppetti P, Nassini R, Materazzi s, Benemei S (2008) The concept of neurogenic inflammation. BJU International 101(3): 2-6.

19. Arai I, Tsuji M, Miyagawa K, Takeda h, Akiyama n, et al. (2015) Repeated administration of IL-31 upregulates IL-31 receptor A (IL-31RA) in dorsal root ganglia and causes severe itch-associated scratching behaviour in mice. Exp Dermatol 24(1): 75-78.

20. Storan ER, O'Gorman SM, McDonald ID, Steinhoff M (2015) Role of cytokines and chemokines in itch. Handb Exp Pharmacol 226: 163-176.

21. Nattkemper LA, Martinez-Escala ME, Gelman AB, Singer EM, Rook AH et al. (2016) Cutaneous T-Cell Lymphoma and Pruritus: The Expression of IL-31 and its Receptors in the Skin. Acta Derm Venereol 96: 894-898.

22. Mishra SK, Hoon MA (2013) The cells and circuitry for itch responses in mice. Science $340(6135): 9$ 68-971.

23. Kardon AP, Polgár E, Hachisuka J, Snyder LM, Cameron D, et al. (2014) Dynorphin acts as a neuromodulator to inhibit itch in the dorsal horn of the spinal cord. Neuron 82(3): 573-586

24. Bourane S, Duan B, Koch SC, Dalet A, Britz O, et al. (2015) Gate control of mechanical itch by a subpopulation of spinal cord interneurons. Science 350(6260): 550-554.
25. Shiratori-Hayashi M, Koga K, Tozaki-Saitoh H, Kohro Y, Toyonaga H, et al. (2015) STAT3-dependent reactive astrogliosis in the spinal dorsal horn underlies chronic itch. Nat Med 21(8): 927-931.

26. Zhang Y, Dun SL, Chen YH, Luo JJ, Cowan A, et al. (2015) Scratching activates microglia in the mouse spinal cord. J Neurosci Res 93(3): 466474 .

27. Davidson s, Zhang X, Khasabov SG, Moser HR, Honda CN, et al. (2012) Pruriceptive spinothalamic tract neurons: physiological properties and projection targets in the primates. J Neurophysiol 108: 1711-1723.

28. Bin Saif GA, Papoiu ADP, Banari L, McGlone F, Kwatra SG, et al. (2012) The pleasurability of scratching an itch: a psychophysical and topographical assessment. Br J Dermatol 166(5): 981-985.

29. Mochizuki H, Tanaka S, Morita T, Wasaka T, Sadato N, et al. (2014) The cerebral representation of scratching-induced pleasantness. J Neurophysiol 111(3): 488-498.

30. Vierow V, Fukuoka M, Ikoma A, Dorfler A, Handwerker HO, et al. (2009) Cerebral representation of the relief of itch by scratching. J Neurophysiol 102: 3216-3224.

31. Jeong KY, Kang KH (2015) Investigation of the pruritus-induced functional activity in the rat brain using manganese-enhanced MRI. J Magn Reson Imaging 42: 709-716.

32. Vierow V, Fukuoka M, Ikoma A, dorfler A, Handwerker HO, et al. (2015) Cerebral networks linked to itch-related sensations induced by histamine and capsaicin. Acta Derm Venereol 95: 645-652.

33. Papoiu AD, Nattkemper LA, Sanders KM, Kraft RA, Chan Y, et al. (2013) Brain's reward circuits mediate itch relief a functional MRI study of active scratching. PLoS One 8(12): e82389.

34. Mochizuki h, Papoiu ADP, Nattkemper LA, Lin AC, Kraft RA, et al. (2015) Scratching induces overactivity in motor-related regions and reward system in chronic itch patients. J Invest Dermatol 135: 2814-2823.

35. Chen L, Wang W, Tan T, Han H, Dong Z, et al. (2016) GABA(A) receptors in the central nucleus of the amygdala are involved in pain- and itchrelated responses. J Pain 17: 181-189.

36. Kim J, Pignatelli M, Xu S, Itohara S, Tonegawa S, et al. (2016) Antagonistic negative and positive neurons of the basolateral amygdala. Nat Neurosci 19: 1636-1646.

37. Kim J, Zhang X, Muralidhar S, LeBlanc SA, Tonegawa S, et al. (2017) Basolateral to central amygdala neural circuits for appetitive behaviors. Neuron 93: 1464-1479.

38. Leibovici V, Magora F, Cohen S, Ingber A (2009) Effects of virtual reality immersion and audiovisual distraction techniques for patients with pruritus. Pain Res manage 14: 283-286.

39. Schut C, Mollanazar NK, Kufer J, Gieler U, Yosipovitch G, et al. (2016) Psychological interventions in the treatment of chronic itch. Acta Derm Venereol 96: 550-551.

40. Nakagawa K, Mochizuki h, Koyama S, Tanaka S, Sadato N, et al. (2016) A transcranial direct current stimulation over the sensorimotor cortex modulates the itch sensation induced by histamine. Clin Neurophysiol 127: 827-832. 
ISSN: 2574-1241

DOI: 10.26717/BJSTR.2018.11.002170

Chan Kam Tim Michael. Biomed J Sci \& Tech Res

(C) This work is licensed under Creative

Submission Link: https://biomedres.us/submit-manuscript.php

\begin{tabular}{ll} 
BIOMEDICAL & Assets of Publishing with us \\
RESEARCHES & - Global archiving of articles \\
& - Immediate, unrestricted online access \\
\hline
\end{tabular}

